\title{
Etiologies and risks of splenic decapsulation after endoscopic retrograde cholangiopancreatography: case report and literature review
}

\section{다(1)우우}

\author{
Authors
}

Vijeta Pamudurthy', Raju Z. Abraham², Thomas Betlej ${ }^{3}$, Ashish Shah", Dong Kim5, Brian Sasso ${ }^{4}$, Abraham Chacko $^{4}$

Institutions

1 Department of Medicine, Riverside Medical Center, Kankakee, Illinois United States

2 Department of Medicine, Section of Pulmonology/ Critical Care, Riverside Medical Center, Kankakee, Illinois, United States

3 Department of Pathology, Riverside Medical Center, Kankakee, Illinois, United States

4 Department of Medicine, Section of Gastroenterology, Riverside Medical Center, Kankakee, Illinois, United States

5 Department of General Surgery, Riverside Medical Center, Kankakee, Illinois, United States

submitted 6.9.2017

accepted after revision 15.11.2017

\section{Bibliography}

DOI https://doi.org/10.1055/s-0043-125145 |

Endoscopy International Open 2018; 06: E271-E273

(c) Georg Thieme Verlag KG Stuttgart · New York

ISSN 2364-3722

Corresponding author

Vijeta Pamudurthy, DO, 350 N Wall Street, Kankakee, IL 60901
Fax: +1-815-928-6266

vijetar@gmail.com

\section{ABSTRACT}

Background and study aims Endoscopic retrograde cholangiopancreatography (ERCP) is an invasive diagnostic and interventional procedure used in conditions related to the pancreas and biliary tract. It has a complication rate ranging from $4 \%$ to $10 \%$. Severe complications are few with the most common of them being post-ERCP pancreatitis, postsphincterotomy bleeding, and perforation. A rare, but potentially life-threatening complication of ERCP is splenic injury. We report the case of a 60 -year-old female with choledocholithiasis who sustained splenic decapsulation following ERCP. The exact causes of splenic injury are unknown, although several mechanisms are postulated. A literature review of splenic injuries post-ERCP shows that there are only 3 cases with post-ERCP splenic decapsulation. Our patient is the first one in whom splenic decapsulation occurred without any risk factors or technical difficulties during the procedure. A high index of suspicion for splenic injury is required in any patient who has severe pain, anemia, or hemorrhagic shock after ERCP.

\section{Introduction}

Endoscopic retrograde cholangiopancreatography (ERCP) is an invasive procedure that is used for both diagnostic and therapeutic purposes related to pancreaticobiliary disease. The procedure has a complication rate ranging from $4 \%$ to $10 \%$, and the rate of severe complication from the procedure is low [1, 2]. The most common complication reported is pancreatitis, with others being bleeding, infection, and perforation [1,2]. Rare complications of ERCP include air embolism, pneumothorax, pneumoperitoneum, impaction of a stone retrieval basket, perforation $[3,4]$, portal vein and hepatic artery cannulation [4], duodenal obstruction [4], and cardiopulmonary events [2].
A rare but potentially life-threatening complication of ERCP is splenic injury. There are many accounts of splenic injury after colonoscopy and are well described in the literature, but splenic injury after ERCP is much less frequent. Splenic injury can include subcapsular hematoma $[5,6]$, peri-splenic hematoma [6], laceration $[6,7]$, rupture $[6,8]$, avulsion of splenic vessels $[6,9]$, avulsion of spleen from the capsule $[6,10]$, and direct tearing of the splenic capsule $[6,8]$. The main factors influencing complication rate were the difficulty of the examination, patient comorbidity, and the operator's level of experience [1, $6]$.

We describe a patient who sustained a splenic decapsulation after ERCP requiring laparotomy and splenectomy. 


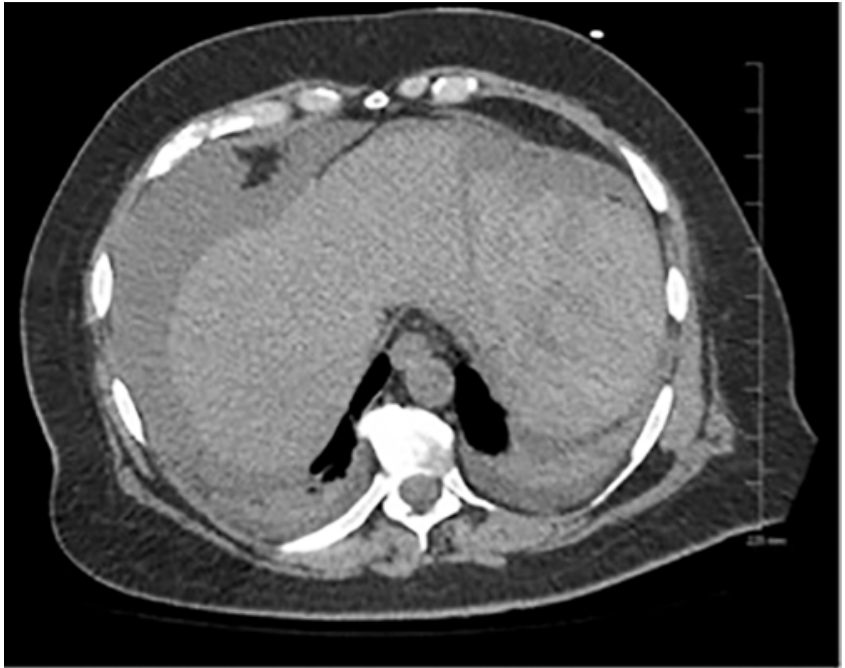

- Fig. 1 CT Abdomen with large left perisplenic hematoma and abdominal ascites

\section{Case Report}

A 60-year-old white female presented with a complaint of sudden onset of sharp, epigastric pain that radiated to the right upper quadrant. The patient underwent an ERCP after there were sudden derangements of liver function tests and evidence of choledocholithiasis on magnetic resonance cholangiopancreatography (MRCP).

During the ERCP the endoscope was passed easily into the second portion of the duodenum, and negotiated into the short position with the ampulla of Vater readily visualized. A 0.035 -inch guide wire followed by a standard sphincterotome was initially passed into the pancreatic duct. The wire tip extended into the body of the pancreas, and it was left in place to help facilitate a second guide wire into the common bile duct. Contrast injection confirmed a small distal filling defect. A small 5-mm sphincterotomy was performed and multiple balloon sweeps removed small amounts of biliary debris. Occlusion cholangiogram was normal. A $5 \mathrm{Fr} \times 5 \mathrm{~cm}$ pancreatic duct stent was placed $4.5 \mathrm{~cm}$ into the pancreatic duct as prophylaxis for post-ERCP pancreatitis and rectal indomethacin $100 \mathrm{mg}$ was given.

Eight hours after the procedure, the patient complained of sharp epigastric pain radiating to her left shoulder and chest. She was found to be hypotensive with a blood pressure of 94/ 70. Hemoglobin dropped from $12 \mathrm{~g} / \mathrm{dL}$ to $6.3 \mathrm{~g} / \mathrm{dL}$. Computed tomographic scanning (CT scan) of the abdomen showed a large left perisplenic hematoma consistent with acute hemorrhage in the region of the splenic hilum with displacement of the spleen inferiorly. There was also a moderate amount of abdominal and pelvic ascites consistent with hemorrhage ( $\triangleright$ Fig. 1). The patient underwent an emergent laparotomy. An estimated $3 \mathrm{~L}$ of blood and clots were removed from the abdomen and showed a decapsulated spleen with splenic rupture. A splenectomy and cholecystectomy were performed. Spleen

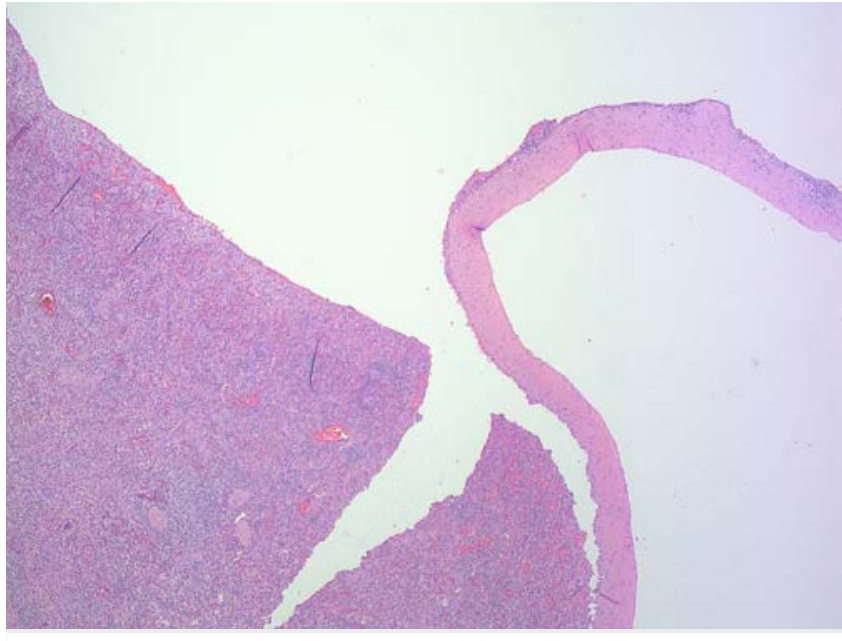

Fig. 2 Splenic decapsulation (H\&E, 100X)

was sent for a pathology review, which confirmed the splenic capsular avulsion ( $>$ Fig. 2 ).

The patient made a full recovery and was discharged 11 days after the initial ERCP.

\section{Discussion}

Based on our literature review, there are a total of 24 reported cases of splenic injury sustained as a complication of ERCP. Ours is the third case of splenic decapsulation following ERCP. In the first case, the patient had multiple risk factors including previous abdominal surgeries and required lysis of adhesions. Symptoms resulted 60 hours after the procedure, and splenectomy was performed [9]. In the second case, the patient had post-ERCP pancreatitis, the procedure was considered to be difficult, symptoms resulted 15 hours after the procedure, and splenectomy was performed [6]. Ours is the first case in which the patient had splenic decapsulation despite the fact that there were no potential etiological factors or technical difficulties with the procedure. Symptoms resulted 8 hours after the procedure, and splenectomy was performed.

There are several risk factors and predictors of occurrence that have been proposed in previous case studies describing splenic injury post-ERCP. Most common and consistent cause of splenic infarction post-ERCP is chronic pancreatitis, which leads to calcification and fibrosis between the supporting ligaments including splenocolic and gastrosplenic ligaments [7]. Excessive traction of the ligaments can lead to mechanical tension and reduced mobility between spleen and surrounding organs $[6,7]$, ultimately leading to rupture [6]. Adhesions from previous abdominal surgeries is also a risk factor for the same reason [6].

Another explanation for ERCP causing splenic injury includes bowing of the endoscope with torsion on the greater curvature of the stomach in the long position, especially while attempting to pass the endoscope through the narrowed duodenum or to cannulate the papilla $[6,9]$. This maneuver translates into direct forces in surrounding organs including the spleen causing 
capsular tears or vascular avulsion. In addition, increased manipulation of the endoscope resulting from prolonged procedure transmits excessive torque to the patient and increases risk of excessive ligamentous tension and may cause splenic injury $[6,8,9]$. Although many causes have been proposed, the true mechanism of post-ERCP splenic injuries remains an enigma [6].

Onset of symptoms in patients with post-ERCP injury can vary widely. While some patients complain of symptoms immediately after the procedure $[8,6]$, it is possible for a patient to report symptoms more than 5 days after the procedure [6]. Based on previous case reports, the most common signs and symptoms include abdominal pain, hemodynamic instability, and a drop in hemoglobin.

Splenectomy may not be necessary for all patients. Conservative management may lead to complete recovery [6]. Angiographic embolization is currently considered to be a part of non-operative management. Splenic artery embolization is indicated in hemodynamically stable patients or patients that are considered easy to stabilize, and it may be used as a bridging therapy for splenectomy [10].

\section{Conclusion}

Splenic injury is a rare complication of ERCP. Although most patients recover completely, that complication can be fatal. It is important to consider splenic injury in any patient after ERCP who has increasing abdominal pain, develops anemia or severe hypotension. These symptoms can present immediately or even days after the procedure. It is important to realize that this complication may occur even if ERCP was performed with no technical difficulty or risk factors. Ours is only the third case with a post-ERCP splenic avulsion from the capsule and it is the first case in which patient had a splenic avulsion from the capsule without any risk factors or technical difficulties during the procedure. The injury can have a delayed presentation, and severity of the injury can be varied. Therefore, a high clinical index of suspicion is essential for providing these patients appropriate treatment after an ERCP.

\section{Competing interests}

None

\section{References}

[1] Suissa A, Yassin K, Lavy A et al. Outcome and early complications of ERCP: a prospective single center study. Hepatogastroenterology 2005; 52: $352-355$

[2] Cotton PB, Garrow DA, Gallagher J et al. Risk factors for complications after ERCP: A multivariate analysis of 11,497 procedures over 12 Years. Gastrointestinal Endoscopy 2009; 70: $80-88$

[3] Chavalitdhamrong D, Donepudi S, Pu L et al. Uncommon and rarely reported adverse events of endoscopic retrograde cholangiopancreatography. Dig Endosc 2014; 26: 15 - 22

[4] Kwon Cl, Song SH, Hahm KB et al. Unusual complications related to endoscopic retrograde cholangiopancreatography and its endoscopic treatment. Clin Endosc 2013; 46: 251 - 159

[5] Montenovo M, Javed E, Bakthavatsalam R et al. Splenic Subcapsular Hematoma After Endoscopic Retrograde Cholangiopancreatography in a Liver Transplant Recipient: Case Report and Literature Review. Exp Clin Transplant 2017; 15: $103-105$

[6] Lee R, Huelsen A, Saad N et al. Splenic Injury Following Endoscopic Retrograde Cholangiopancreatography: A Case Report and Literature Review. Case Rep Gastroenterol 2017; 11: 241 - 249

[7] Gaffney RR, Jain V, Moyer MT. Splenic Injury and ERCP: A Possible Risk for Patients with Advanced Chronic Pancreatitis. Case Rep Gastroenterol 2012; 6: $162-165$

[8] Geddoa E, Qureshi NA, Patten DK et al. Splenic Laceration After Endoscopic Retrograde Cholangiopancreatography. CRSLS; 2014: $1-6$

[9] Zyromski N], Camp CM. Splenic injury: a rare complication of endoscopic retrograde cholangiopancreatography. Am Surg 2004; 70: $737-739$

[10] Wu WC, Katon RM. Injury to the liver and spleen after diagnostic ERCP. Gastrointest Endosc 1993; 39: 824-827 